\title{
Trivet Fields: The Materiality of Interaction in Architectural Space
}

\author{
Dagmar Reinhardt \\ and Joanne Jakovich
}

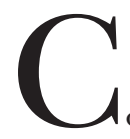

onventionally, the materiality of architectural space exists in the collective effect of the visual, formal, acoustic and tactile qualities of the built materials under certain conditions of light, situation and time [1]. It is the sum of the perceived space, not the materials or objects in isolation. Architectural design is the process of specifying articulations and variations in materials within these conditions for the purpose of satisfying and potentially influencing human behavior and experience in the space [2]. This implies that traditional architectural design anticipates, and arguably orchestrates, human interaction in and with the space, through materiality.

Interactive technology introduces a new dimension to this relationship, i.e. behavior within the space that is not only an outcome of design but may also be used to drive dynamic architectural elements in real time: interactive walls/doors/ floors [3], operative modular surfaces [4], reconfiguring wet surfaces [5], media projections [6], dynamic furniture [7], digital soundscapes [8], ambient info-displays [9] and so forth. The pioneering work by interactive artist Myron Krueger in the early 1970s was the first to establish aesthetic materiality of interaction [10]. Krueger developed a series of "responsive environments" in which the audience could use full body

Dagmar Reinhardt (architect, educator), Faculty of Architecture, Design \& Planning, University of Sydney, NSW 2026 Australia. E-mail: <reinhardt5@yahoo.com>.

Joanne Jakovich (architect, educator), School of Architecture, Faculty of Design, Architec ture and Building, University of Technology Sydney, Ultimo NSW 2007 Australia. E-mail: $<$ Joanne.Jakovich@uts.edu.au>.URL:<http://jakovich.net>.

Based on a paper presented at the sixth Creativity and Cognition conference (13-15 June 2007, Washington, DC), on the theme "Materialities for Creativity," focused on the cultivating and sustaining of creativity.

Article Frontispiece. Trivet Fields, modular sensate installation, 2006. ( Alex Jung, Dagmar Reinhardt, Joanne Jakovich, Phil Granger) An adaptive Perspex sensory machine constructed from interlocking modules that host a field of sensors and audio-illuminate displays. The work's sensate memory system builds on the interactions from visitors over time. gestures to interact with an array of spatially projected digital media. He discovered that the composition of the relationships between action and response drove the aesthetic experience, while the beauty of the visual and aural displays were secondary. Notably, Krueger proposed "response" as a "new art medium based on real-time interaction between men and machines" [11], bringing to light the potential for interactivity itself as a design element articulating space that is able to evoke a sensual and tangible experience. In a similar way, interactive sound artist David Rokeby describes the "construction of experience" as the creative goal of the artist and argues that the content of the artwork lies in the interactive experience itself [12] over any physical or symbolic representation.

Furthermore, contemporary computation allows the deconstruction of the action-response cycle into infinite permutations of interactions over time in space [13]. Human activity, captured by sensors or computer vision, can be translated and interpreted using models of basic cognition [14] and can also inform generative processes of expression that produce sophisticated, multidimensional responses built up over time with numerous users [15]. The infinite variation of such computational structures, in combination with the articulations of matter as interface (light, form, material), forms what was previously an unfathomable palette of interactive "materials" for contemporary spatial and interactive design.

In light of this new aesthetic potential, we recognize the requirement to reconstruct the basic conceptual framework of architecture to accommodate the materiality of interaction, extending from sensor-based devices to material structures. Ar-

Fig. 1. Drafting processes of the Perspex modules comprising the Trivet Fields installation. (๑ Alex Jung, Dagmar Reinhardt, Joanne Jakovich, Phil Granger) The modules were laser cut and heat molded to allow the prongs to act as interconnecting nodes in 3-dimensional space.
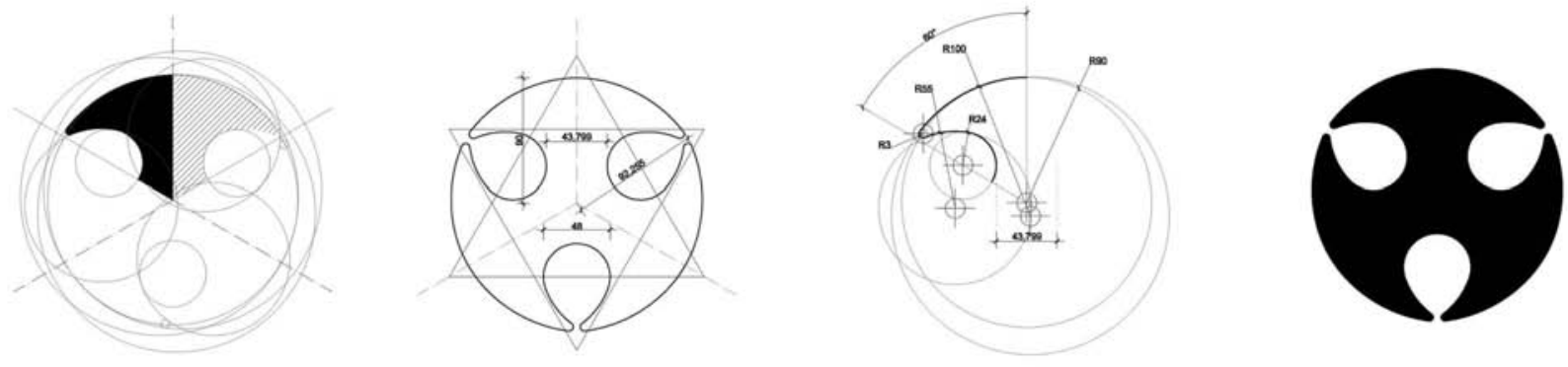


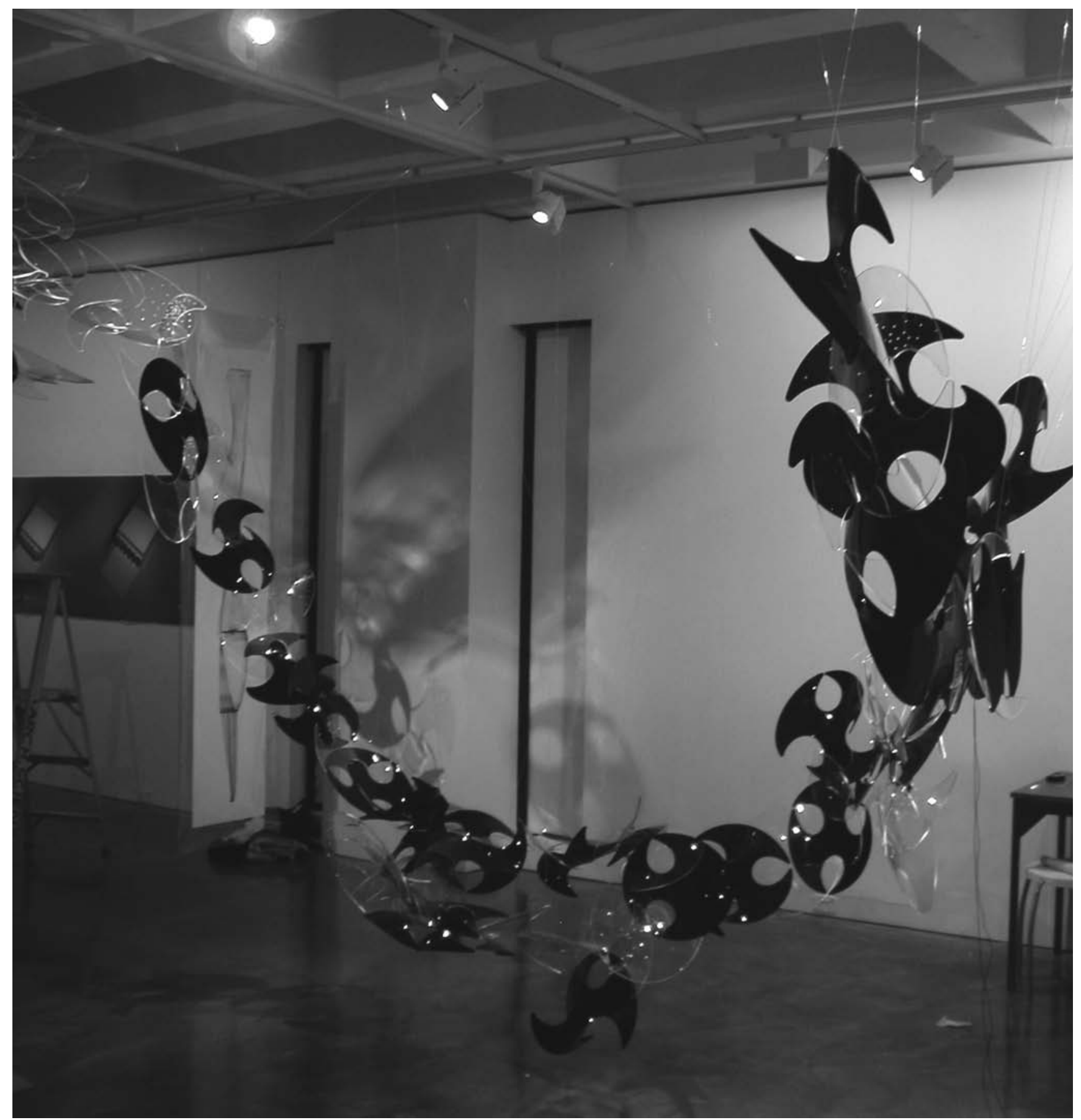

Fig. 2. Trivet Fields, modular sensate installation, 2006. (๑ Alex Jung, Dagmar Reinhardt, Joanne Jakovich, Phil Granger) One configuration of Trivet Fields, using about 35 modules, on display at the Tin Sheds Gallery, Sydney, October-November 2006.

chitectural practice might then develop a vocabulary of space that incorporates the indeterminate, interrelated dimensions of interactivity meshed with an expanded understanding of the perceptual and generative qualities of physical materials. Through our creative practice, spanning interactive art and architecture, we explore the aesthetic potential of this paradigm, reconsidering concepts from architecture and interactive art in order to develop an understanding of the materiality of interaction in space. The following outlines a spatial paradigm and its sub-concepts of architecture, interaction and materiality. We demonstrate these ideas in a recent installation work, Trivet Fields [16], that explores the integration of physical and interactive mechanisms to articulate spatial materiality.

\section{RECONSIDERED: \\ ARCHITECTURE, MATERIALITY AND INTERACTION}

While architecture defines space, it may also be described as a system of interacting and interrelated components [17]. Such a system has a specific structure that presets interrelationships between its component parts and organizes the behavior of the system overall. Its structure may vary in interactive capacity, from fixed, responsive, adaptive, to autonomous properties, depending on the individual characteristics of its component parts. Architectural structures are determined by material components, yet architecture is also concerned with human interaction with the constructed systems. Hence, the components interplaying in this system are equally comprised of physical parts, elements of interactive technology and the human inhabitant [18]. Consequently, a para- 
digm that reconfigures a new materiality of interaction in architectural space consists of the subjective mapping from elements of human experience to elements of other components of the system. Once implemented, the system thus adopts and adapts to relationships initially specified, between patterns of human or material component behavior.

Materiality and its effects emerge from the interactive properties of architectural space. While a material system follows a given structure, its materiality may negotiate a more transient state. A material might translate between intermediate conditions of two or more states, such as brightness of reflection and dullness of sound. The medium's main characteristics lie in its inherent capacity of transition, or transformation. Through its changing performance, a material acts as a method for exchange, as a device for continual creative exploration. Dynamic changes or effects such as in surface characteristics, light conditions, spatial atmosphere or program sequences thus iteratively determine the material- ity of a system's component parts. The trigger can be an interiorized program signal (digital or electronic circuit), a direct reaction to user contact (visual, tactile) or a registered shift in ambient condition (light, temperature, currents). Transitional stages, spatial effects and behavioral potential can thus be expressed according to the shared affordances and constraints of the materiality experienced over time.

In an architectural system, interaction may be described as the combined reciprocal perception and action between two or more component elements that have an effect on each other in a spatial, contextual and temporal way [19]. Components in the architectural system may be of human, material (hardware, building part) or digital (software, sensor system) character, yet with a capacity for interacting in a non-predetermined manner. Components possess a means for receiving information from others and for expressing (displaying) information in return. Thus, interaction produces feedback as direct and implicit informa- tion about how actions are interpreted by related components. The materiality of interaction is not only informed by material effects but equally by a feedback loop that occurs directly through reciprocated action or is indirectly passing through the overall effect of system actions. This dialogue in architectural space develops as specific to that context and not repetitive, according to data exchange over time between diverse component parts. Interaction here is based on temporal accounts that can be cross-referenced and thus can produce generalized rules about the causes and effects of actions.

In this manner, architectural system, materiality and interaction constitute a new spatial paradigm that is reconfigured according to the shared properties and affordances of its system members.

\section{RELATED MODULAR INSTALLATION WORKS}

Recent interactive installations such as works by Decoi [20], Nox [21], Beesley

Fig. 3. Trivet Fields, modular sensate installation, 2006. (@ Alex Jung, Dagmar Reinhardt, Joanne Jakovich, Phil Granger) Detail of the variations in form and puncturing of modules, which differ in color, luminosity, reflectivity, punctuation pattern, degree of curvature, registering and emissive capacities.

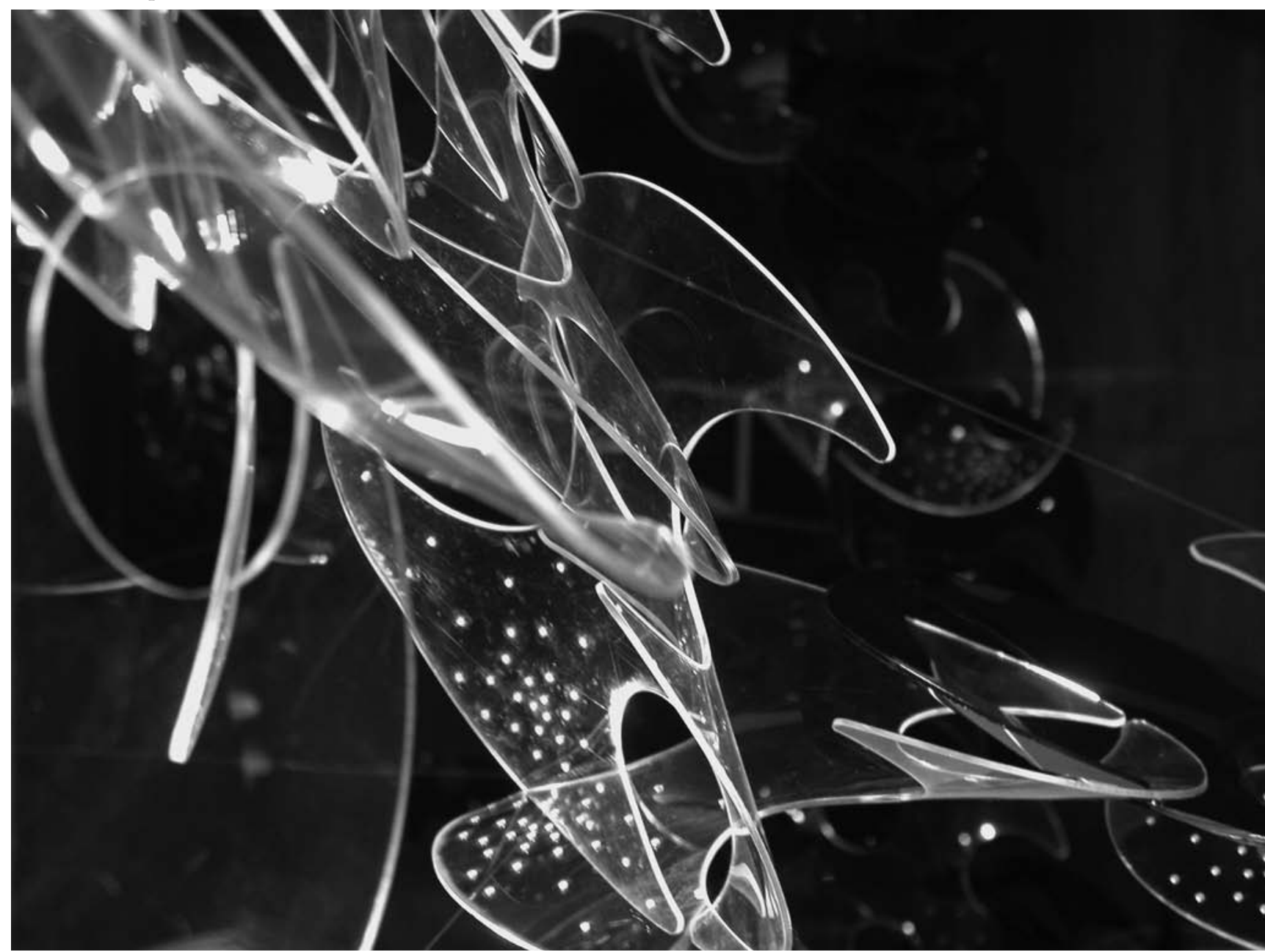




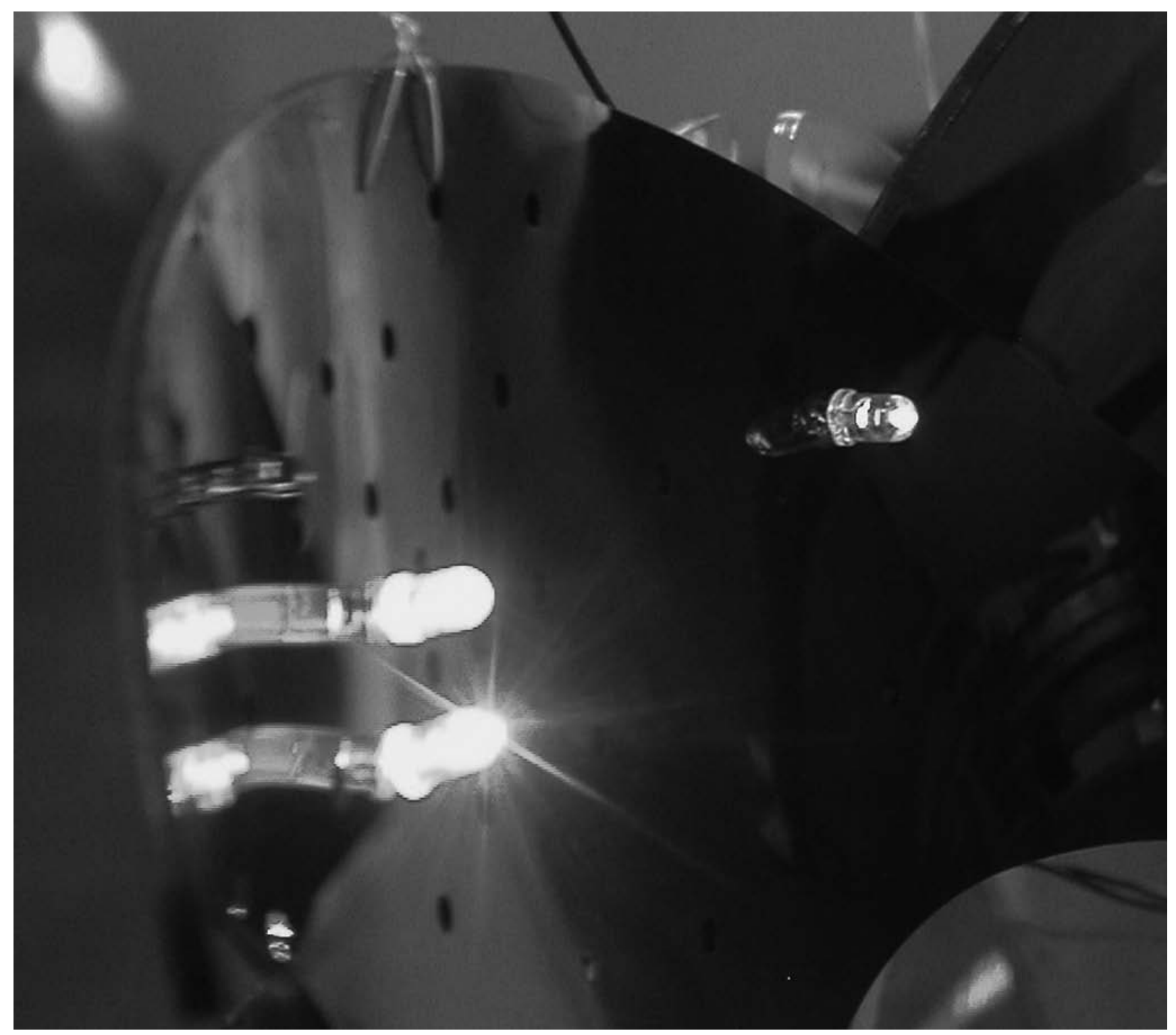

Fig. 4. Trivet Fields, modular sensate installation, 2006. (@ Alex Jung, Dagmar Reinhardt, Joanne Jakovich, Phil Granger) The trivet modules host a series of simple motion sensors, mini-speakers and LED lights.

[22] and Servo [23] utilize dynamic, responsive spatial setups that change in temporalization through interactivity and illustrate a materiality of interaction in architectural space. Yet, while all belong to a genre that bridges interactive installation art and architecture, there are particular differences between their material characteristics, systems structure and component behavior, so in each a unique form of materiality of interaction is defined.

A first type of installation, employing homogenous surface formations, is the repetition of identical or similar modules with equal material properties and behavioral characteristics, as might be said of the installations by Nox or Decoi. Here, many become one; components react as structural entities in relationship to each other. The system responds collectively, through an interaction produced by me- chanical response, or by a shift of ambient conditions. Individual modules may possess the capacity to receive individual information, yet are restricted to a field behavior that illustrates an alteration in overall form or shape. Hence, the channel of communication between event and response is widened and blurred, evoking an ambient materiality generated through pooled activity.

Lattice Archipelogics illustrates a second type of installation. Here Servo uses a spatially distributed installation in a time-based interactive context [24]. This dynamic audiovisual environment uses predominantly virtual media to engage interaction. A hundred identical plastic modules form the interactive field of a sentient space. The modules' cavities house wiring as well as sensory, sonic and light devices. A select number of these components register the prox- imity or movement of visitors and mediate this information to virtual program agents. These are the virtual counterpart of the digital reality, which reflects forces and motion exerted by visitors as ambient media patterns; light and sound are spatial, dynamically interrelated effects exchanged as "clustered immaterial voxels" [25]. While the spatial configuration is complex, the material of the installation remains static and is continuous; its materiality is dynamic through digital conversion between sensed behavior and electronic media.

A third type of installation utilizes distinct component parts that respond on an individual basis. This may be illustrated by the installation Orgone Reef, in which Philip Beesley uses a complex configuration of laser-cut Perspex modules that establish a layered structure [26]. Each module is built from several 


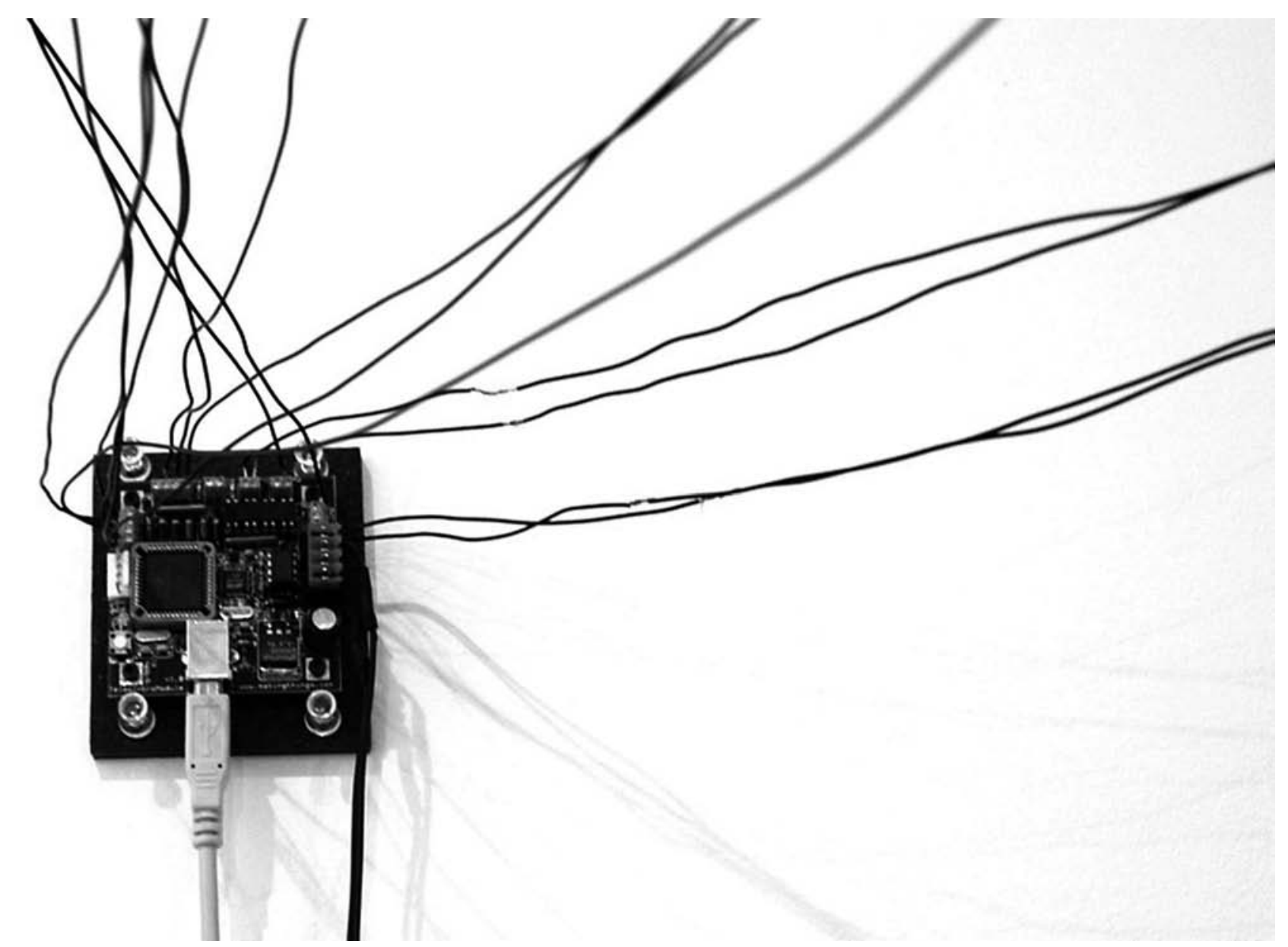

Fig. 5. Trivet Fields, modular sensate installation, 2006. (@ Alex Jung, Dagmar Reinhardt, Joanne Jakovich, Phil Granger) Processing board attached to nearby wall hosting sensor control in-outs. The software component controls the mapping between sensed motion and emitted sound. As users begin to walk past and become aware of sounds emitted from the space, the software gradually adapts to their behavior and new sounds are evolved.

segments that include a structural threedimensional body, whiskers, an injection unit needle and a bladder reservoir. The modules are arranged in the non-repeating geometrical system of a Penrose tessellation, using a minimal amount of raw material that expands in space to form a network of a porous volume. The Orgone Reef structure responds through vibration and oscillation to real-time changes such as air currents produced by ambient streams or visitor movement. It is modeled not as a constructed surface, but as an animated collection of nodes evoking the hybrid ecology of an artificial nature that submits to natural processes, such as sedimentation, blending and dissolution. It engages the visitor with "large-scale field structures [that] offer bodily immersion and create a wide-flung dispersal of perception" [27]. The reef system produces a response to the aftereffects of gestural interaction, such as atmospheric shifts, traverse or an interruption of the ambient spatial setting. In that sense, the materiality defined through dynamic input is somewhat reflective of the degree of dispersion of information, enabling both ambient and directed interactive material experiences.

These works demonstrate that, depending on the architectural system, the materiality and characteristics of components and their role of response, a different materiality is generated by interaction, and, likewise, interactivity is instigated by the interstitial nature of temporal and material dimensions of the system.

\section{TRIVET FIELDS: IMPLEMENTING MATERIALITY OF INTERACTION IN ARCHITECTURE}

With respect to recent interactive works as discussed previously, we examine our work in which particular considerations, characteristics and potentials of interactive materiality are applied in the experimental set-up of a tactile interactive space. Trivet Fields is a responsive, spatially adaptable installation constructed from interlocking modules that host a field of sensors and audio-illuminate displays, acting on a sensate memory system that builds on the interactions from visi- tors over time [28]. It is a collaboration between the authors and Alexander Jung and Philip Granger, merging the skills of architecture, interaction design and digital fabrication. It is both a physical and interactive solution to the architectural question of spatial delineation (Article Frontispiece). Trivet Fields might be inserted into an existing host architecture in different configurations. The interlocking field is suspended by poly wire in several points and attached to the host space's defining boundaries. The system is thus detached from structural, functional and programmatic constraints but rather defines a territory differentiated from its surrounding spaces by a zone of attention generated through animated matter.

In Trivet Fields, a variable number of laser-cut and heat-molded modules (80-110 pieces) are arranged to create a continuous, reconfigurable field. While other interactive installations consist of identical modules that produce a homogenous arrangement, we use a surface materiality that differentiates zones and intensities through individuals and 


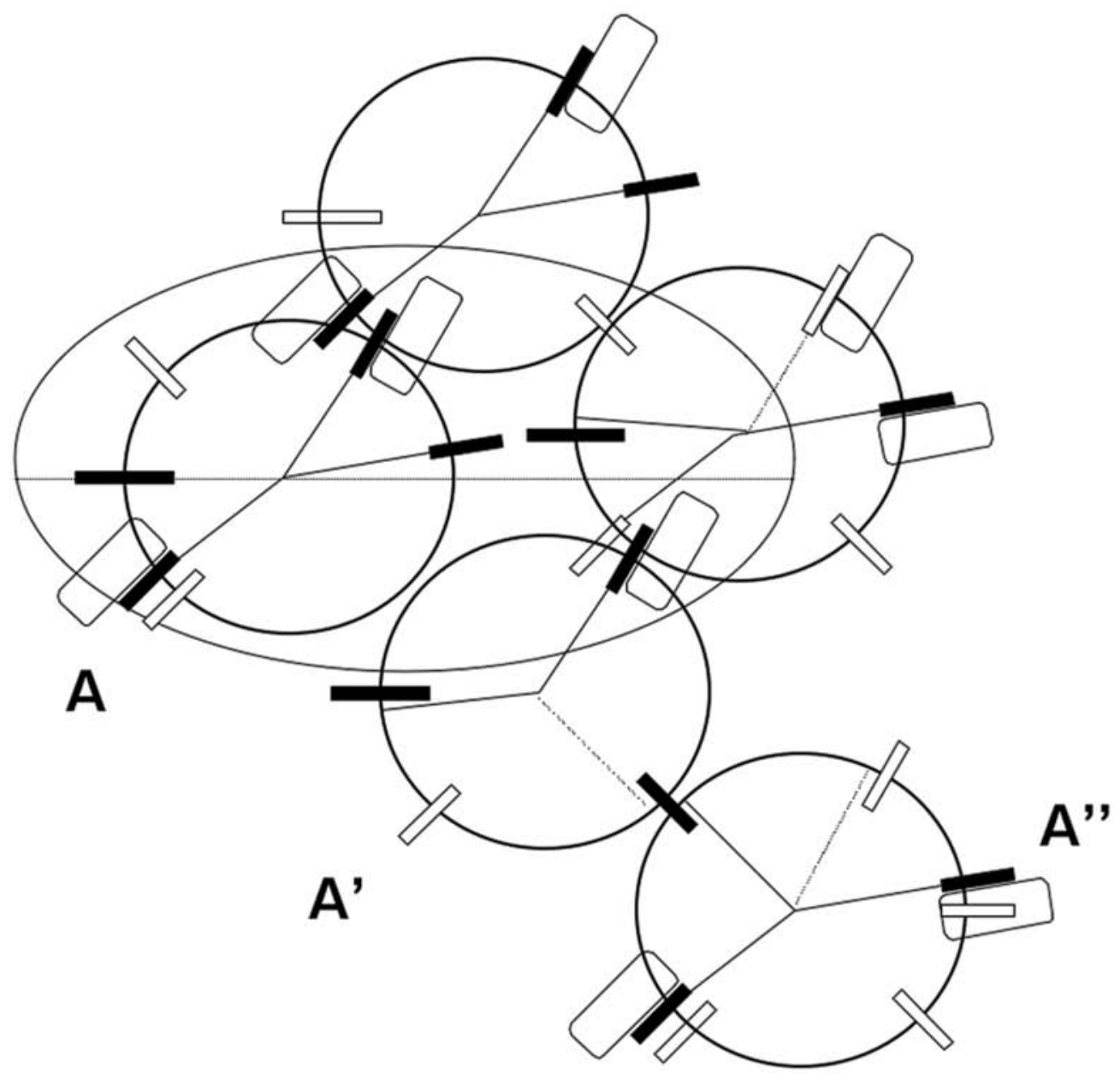

Fig. 6. Trivet Fields, modular sensate installation, 2006. (@ Alex Jung, Dagmar Reinhardt, Joanne Jakovich, Phil Granger) Diagram of a component group of the Trivet Field, with diverse behaviors through combinations, reconfiguring through the software nodes over time.

specimen groups that display a range of characteristics. The material character of each Perspex component determines a possible role, position and also a responsive behavior in the field-toward other components and toward the visitor. The modules differ in color, luminosity, reflectivity, punctuation pattern, degree of curvature, registering and emissive capacities. Depending on color-clear or black-the modules reflect or transmit ambient light or cast shadows, focus and intensify light on their exterior contour line or display reflections from other modules' surface parts.

The trivet (3-prong shape) is based on a non-directional figure that allows for a series of configurations (Fig. 1). From a two-dimensional form, two formal variations of modules are generated. The first are heat-molded in a subtle precise soft curvature over a half sphere to form interconnected nodes. The second receive an approximate individual curvature in which all three pods are deformed in xyz direction to form the expansive modules. The deformation thus defines an interlocking capacity within the field, a property of assembling or extending the surface figure further into space (Fig. 2).

According to a pattern imprinted during fabrication, the modules vary in surface texture through a punctuation of holes (Fig. 3). The pattern structure also receives devices of interactive computing; its materiality determines a tactile and visual trigger to engage with, to activate the installation. The modules host a series of simple motion sensors, mini-speakers and LED lights that are wired up to a central in-out board (Figs $4,5)$. The software component controls the mapping between sensed motion and emitted sound. Initially the sensoraudio points of the installation are configured as nodes in a self-configuring, fully connected network. The motion of an approaching individual activates the network. A relationship between the human user and the digital and material components in the dynamic environment is activated through acoustic, sensory and light transmitting elements and transferred to a digital realm in which agents act as communicators in a hybrid zone conflating physical and immaterial world (Color Plate E). The network gradually adapts the weighting between nodes, such that nodes begin to emulate sounds and reactivity patterns of their strongest neighbors. Each node develops sounds that change over time and in this way the work is intended to engage the user in an ongoing state of reflective activity. When the installation is in a no-input condition, it pursues its own path and emits low-level ambient sounds.

This network of reconfigurable sensory components, expressive modalities, human agents and material formations operates by integrating diverse entities in a field condition or territory that respects the distinctiveness of each and allows individual response and relationship interaction. Such field conditions are based on a "network of relations capable of accommodating difference, yet robust enough to incorporate change without destroying its internal coherence" [29]. In order to be responsive, experiential and adaptable, a heterogeneous component system needs to submit to principles, 
behavior and relative distances. Random encounters form a pattern that delivers a meta-information of group behavior, through which individual transfer and transactions form the primary mechanism establishing and administering territory and content.

The materiality of interaction that forms the Trivet Fields is hence based on the dynamics of organization (Fig. 6). Instead of homogeneous systems or a stable organizational figure, it employs the territorial condition of its surrounding space and the internal consistency of diverse component formation and interaction in use. Form, absence of form, edge conditions, texture and shadow define an environment of dynamic relationships, in which not only materiality, but also the non-materiality (the voids in between), define spatial and behavioral potential (Fig. 7). Architectural space here employs a materiality of interaction that might be described as a Deleuzian passage of becoming-becoming other [30]. As we have observed in people's interaction with the installation, individuals tended to enter into a relationship with the material structure in which they explored light and sound responses through gestural interaction. The evolving sound fragments, changing reflections and light transformations observed emerge as an ephemeral spatio-temporal setting that seemingly connects to a domain of the subconscious or irrational, evoking a deep state of engagement. In this way the installation opens an operative experiential field, in which for a moment an immediate perceptual plane might be produced [31].

\section{CONCLUSIONS}

Our creative explorations have initiated a discussion on the role of interaction in spatial materiality. Trivet Fields is one example from a series of installation works that introduce interactive art computing to architectural practice, providing a new platform for cross-disciplinary explorations in materiality of space. Here spatial interaction is a result of programmed elements, material effects, component organization and also the framework and interstices between modules. In turn, spatial form operates in a non-determined behavioral field through an informed notion of interactive materiality.

We suggest that such installations can create a passage and a territory in which space is perceived as sensation, as a state with potentiality. Neither centered around devices or programming systems, nor predetermined by material structures, the emergent outcome of an animated materiality is the generation of sensation. The body enters a relationship with dynamic materiality, through which a genuine experience is generated. Each time the sensation is immediate and individual. The interaction organized and processed in such a material field produces a zone of attention and affects a surface formation that continuously interpolates between movement, figure and field. Hence it could be suggested that matter becomes dynamic through

Fig. 7. Trivet Fields, modular sensate installation, 2006. (@ Alex Jung, Dagmar Reinhardt, Joanne Jakovich, Phil Granger) Space is articulated by form, absence of form, edge, texture and shadows. The Field defines a territory differentiated from its surrounding spaces by a zone of attention generated through animated matter.

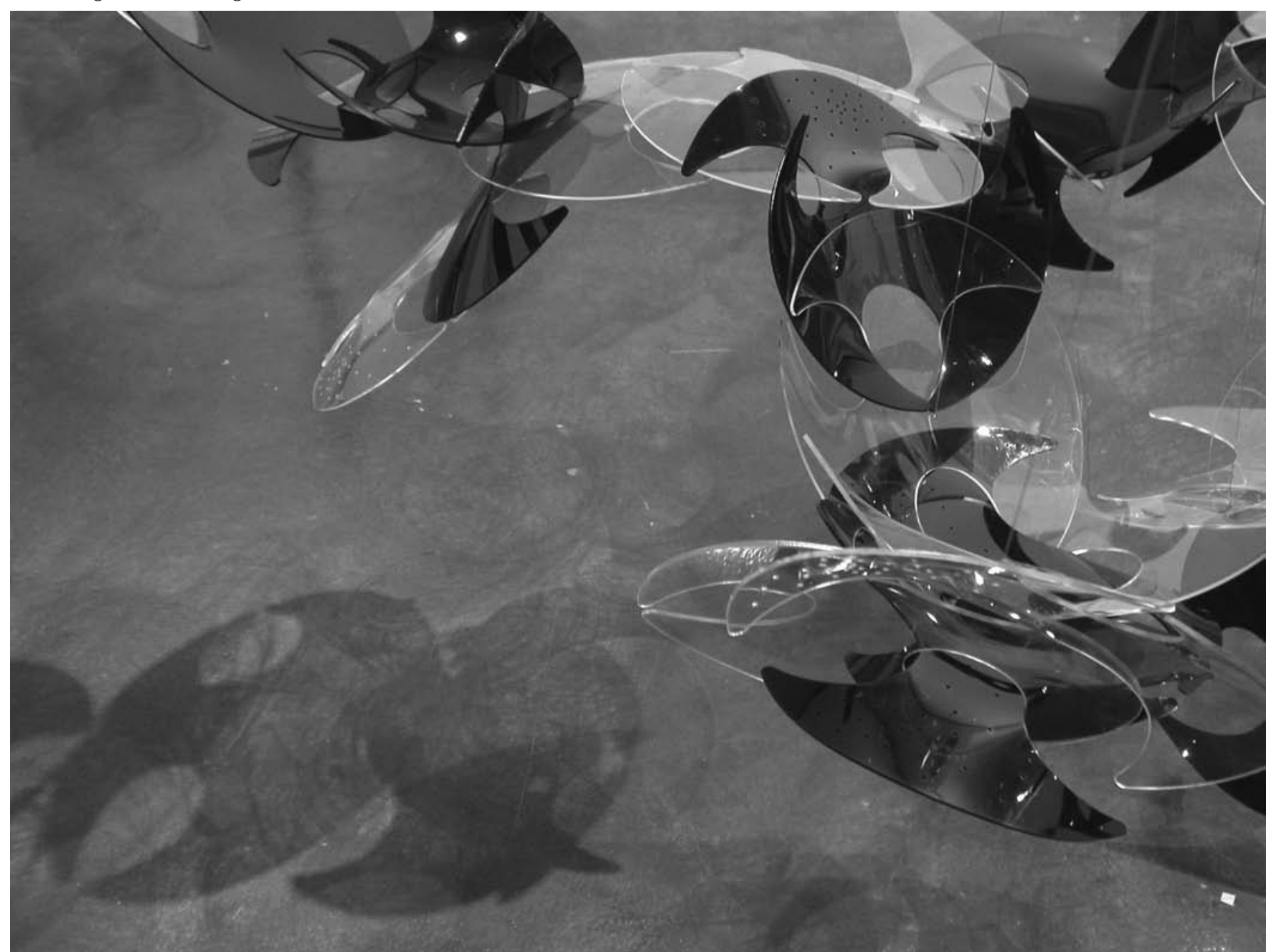


its effects on perception, in addition to perception being influenced by matter. Matter or material, then, must be conceived both in terms of its capacity to express as well as its capacity to instigate expression. That is, materiality is the interface inviting and enabling a variety of modes of interactivity, as well as the medium through which response is communicated.

We have attempted to reconstruct the basic conceptual framework of architecture to accommodate the materiality of interaction, extending from sensor-based devices to material structures. We intend this to be used in architectural practice to develop a language of space that incorporates the indeterminate, interrelated dimensions of interactivity and the perceptual and generative potential of basic architectural materials. The specificity and effects of the suggested new generation of interactive installation architecture hence depend on combined material component properties and reactive behavioral capacity towards the user, thus effectively establishing a new paradigm of architectural materiality. Our aspiration is to feed back into architectural practice and theory through practical experiments and reflective practice.

\section{Acknowledgments}

This article is based on the poster-demonstration paper presented at the 6th Creativity and Cognition paper presented at the 6th Creativity and Cognition
Conference, June 2007 in Washington DC: "SonicConference, June 2007 in Washington DC: "Sonic-
tecture: Esthetic Spatial Conditioning Through tecture: Esthetic Spatial Conditioning Through
Sound, Computation And Interaction," in Creativity and Cognition: Seeding Creativity-Tools, Medi and Environments (Washington, DC, USA: CC2007 Conference Proceedings, 2007), pp. 255-256.

This research is supported by a University of Sydney Postgraduate Award, a Cooperative Research Center for Construction Innovation Scholarship and an Endeavour International Postgraduate Research Scholarship at the University of Sydney.

\section{References and Notes}

Unedited references as provided by the authors.

1. Zumthor, Peter, Atmospheres: architectural environments; surrounding objects (Basel: Boston: Birkhäuser, 2006)

2. Lawson, Bryan, The language of space, (Oxford; Boston: Architectural Press, 2001).

3. Garcia, Mark, Otherwise Engaged: New Projects in Interactive Design, in Architectural Design, Volume 77, Issue 4, Date: July/August 2007, Pages: 44-53. For example, the Realities United "SPOTS" project on Potzdammer Platz in Berlin, p.50.

4. Mark Goulthorpe, The Possibility of (An) Architec- ture (New York: Routledge, 2007). For example, the modular smart wall "Aegis Hyposurface," <http:// www.sial.rmit.edu.au/Projects/Aegis_Hyposurface. php>.

5. Lars Spuybroek, NOX: machining architecture (London: Thames \& Hudson, 2004). For example, Nox's "Waterpavillion" built for the H2Oexpo, The Netherlands (1993-1997).

6. Lucy Bullivant, Responsive environments: arch tecture, art and design (London: V \& A, Inc., 2006) For example, Rafael Lozano-Hemmer's "Bodymovies," plaza installation in Rotterdam, <http:// www lozano-hemmer.com/video/bodymovies.html>.

7. Omojola, Olufemi, et al., An installation of interactive furniture, in IBM Systems Journal 39(3\&4) $861-879,2000$

8. Christian Moeller, A time and place: Christian Moeller, media architecture 1991-2003 (Baden, Switzerland: Lars Müller Publishers, 2004). For example, "On Air" interactive sound installation, Castle Eggenberg in Graz, April 2003, <http://www.christianmoeller.com/display.php?project_id=37>.

9. Xiaobin Shen, et al., Intrusive and Non-intrusive Evaluation of Ambient Displays, Issues in the Design and Evaluation of Ambient Information System Workshop 2007, Toronto, Canada, pp. 30-35.

10. Myron Krueger, Responsive Environments, in Randall Packer and Ken Jordan (eds.), Multimedia: From Wagner to Virtual Reality (New York, US: Norton \& Company Ltd, 2001), pp. 104-120.

\section{Krueger [10].}

12. David Rokeby, The Construction of Experience, in: Clark Dodsworth (ed.), Digital Illusion: Entertaining the Future with High Technology (New York, US: ACM Press, 1998)

13. John McCormack, Art and the Mirror of Nature, Digital Creativity 14(1):3-22, 2003.

4. Michael Walter, et al., Auto-clustering for unsupervised learning of atomic gesture component using Minimum Description Length, Proc. 2001 IEEE ICCV Workshop on Recognition, Analysis and Tracking of Faces and Gestures in Real-time Systems, Vancouver, Canada, pp. 157-163.

15. McCormack [13]

16. <http://jakovich.net/trivet $>$

17. Joanne Jakovich and Kirsty Beilharz, From Audience to Inhabitant: Interaction as a Medium in A chitecture, ENGAGE: Interaction, Art And Audience Experience (Sydney, Aus: UTS, 2006), pp. 40-49.

18. Jack Burnham, Systems Esthetics, Artforum 7(1):30-35, 1968.

19. Jakovich \& Beilharz [17].

20. Goulthorpe [4].

21. Spuybroek [5].

22. Philip Beesley, Orgone Reef, Installation in Winnipeg, Manitoba (2003), published in: Design Through Making, Bob Sheil (ed.), Architectural Design, AD
75(4): 46-53 (London: Wiley Academy, 2005). See also: Philip Beesley, <http://www.philipbeesleyarchitect.com/sculptures/0126manitoba_orgone/orgoneinfo.html >.

23. Servo and Smart Studio, "Lattice Archipelogics," Installation for Latent Utopias (2002), access date: 070319 , <http:/ /www fundacion telefonica.com/at/ vida/paginas/v5/esmart.html > and <http://smart. vida/paginas/v5/esmart.html> and <http://smart. tii.se/smart/projects/lattice/index_en.html $>$ and
Servo-Lattice Archipelogics, in: Architectures Non
Standard, Exhibition Catalog (Paris: Centre Pompidou, 2004), pp. 180-181.

24. Servo and Smart Studio [23].

25. Bullivant [6]

26. Beesley [22].

27. Beesley [22]

28. Dagmar Reinhardt, Alexander Jung, Phil Granger and Joanne Jakovich, Trivet Fields, Velocity Exhibition, The Tin Sheds Gallery (2006); Sydney and Joanne Jakovich and Dagmar Reinhardt, Sonictecture: Esthetic Spatial Conditioning. Through Soure Esthetic Spatial Conditioning Through ene and Cognition. Seeding Creativity-Tools, Media and Environments (Washington, DC, USA: CC2007 Conference Proceedings, 2007), pp. 255-256.

29. Stan Allen, From Object to Field (London: Architectural Design No.127-Architecture after Geometry, 1997), pp. 24-31.

30. Gilles Deleuze, Francis Bacon-The Logic of Sensation (London: Continuum, 2003).

31. Dagmar Reinhardt, Surface Strategies and Constructive Line: Preferential Planes, Contour, Phenomenal Body in the Work of Bacon, Chalayan, Kawakubo, Colloquy/09 (Victoria, Aus: Monash University, 2005), pp. 48-70, <http://www.colloquy. monash.edu.au/issue009/index.html>.

Dagmar Reinhardt is an architect and principal of reinhardt_jung, an office that specializes in architecture research, interactive installations, buildings works and architectural theory, based in Frankfurt/Main and Sydney. Reinhardt received a postgraduate degree of Conceptual Design from Städelschule Frankfurt (University of Fine Arts), where she taught with Ben van Berkel, Peter Cook, Enric Miralles and Mark Wigley. Reinhardt has been the Studio Leader of the Master of Architectural Design at the Faculty of Architecture, University of Sydney, where she is currently completing Ph.D. research that investigates designing duration in contemporary fashion and architecture and focuses on dynamic surfaces and spatial strategies. This work is supported by an IPA and IPRS scholarship from the University of Sydney.

Joanne Jakovich is an interactive architect and researcher specializing in interactive spaces spanning art, architecture and urbanism. Creative outcomes include interactive soundspaces, online urban planning simulations and urban development frameworks. She has exhibited in Japan, Australia, Taiwan and the Netherlands and produced international projects integrating urbanism, architecture and interactive art. She graduated from the University of Tokyo on a Japan Ministry for Education Scholarship and is pursuing a Ph.D. in Architecture at the University of Sydney. She holds a CRC for Construction Innovation Scholarship and University of Sydney Postgraduate Award. She is currently a Senior Lecturer at the University of Technology Sydney. 